\title{
The Construction and Implementation of a Cancer Precision Nursing Model
}

\author{
Xunya Xiong1, Jun'e Hu², Zhaowu Ma1, Xiaochun Peng1* \\ ${ }^{1}$ Department of Medicine, Yangtze University, Jingzhou, China \\ ${ }^{2}$ Oncology Department, Jingzhou Central Hospital, Jingzhou, China \\ Email: `674209597@qq.com
}

How to cite this paper: Xiong, X.Y., Hu, J.E., Ma, Z.W. and Peng, X.C. (2019) The Construction and Implementation of a Cancer Precision Nursing Model. Yangtze Medicine, 3, 1-9.

https://doi.org/10.4236/ym.2019.31001

Received: August 28, 2018

Accepted: December 18, 2018

Published: December 21, 2018

Copyright (c) 2019 by authors and Scientific Research Publishing Inc. This work is licensed under the Creative Commons Attribution International License (CC BY 4.0).

http://creativecommons.org/licenses/by/4.0/

\begin{abstract}
This article reviews the precision nursing of cancer patients from three aspects: concept, system construction and clinical application. It is suggested that nurses should correctly understand the connotation of precision nursing, expand the research content, enrich the research methods, actively carry out the targeted cancer precision nursing practice, improve the quality of life of the patients, and then form the shape of the patient. It is a localized nursing care system that can provide the best medical care for every cancer patient.
\end{abstract}

\section{Keywords \\ Cancer, Precision Nursing, Nursing Practice, Review}

\section{Introduction}

Precision nursing refers to the caregiver's accurate phenotype or the deep phenotype analysis of the patient, and gives the right patient accurate nursing practice at the right time [1]. Cancer precision nursing refers to the combination of gene or gene spectrum analysis of cancer patients with cancer specialist care such as medication compliance, symptom groups, influencing factors and so on, which is a multidisciplinary collaborative care model [1] [2].

Combining the connotation and development of precision medicine and exploring the challenges of precision medicine for clinical care, researchers such as Yan Qiaoyuan pointed out that nursing subjects in the context of precision medical nursing should adopt countermeasures such as precision nursing, precise management, and precision services [3]. Nowadays, the tasks nurses undertake in clinical departments of hospitals are not limited to basic care, but also bear the tasks of expert assistants, cooperation with endoscopic procedures and various examinations, nursing specialist out-patient clinics, psychological coun*Corresponding author. 
seling, triage coordination, and so on [4]. This requires more highly educated nursing professionals to adapt to the development of precision nursing. Furthermore, in the assessment of cancer risk, understanding of the pathogenesis, predicting the best treatment of complications, cancer symptom management and psychological nursing, etc., to promote the maximization of precision medical benefits in many aspects are all important contents of the American Precision Medicine Program [5] [6].

Individual differences in patients will lead to differences in diagnosis and treatment and nursing. For this point of view, nursing staff in different countries have reached consensus. However, the biggest difference in cancer patients is reflected in the patient's genetic or genome variation. Researchers have proposed that combining genetic or genome variation with different patients to provide interventions based on biological research evidence at different stages of the disease has reached potential care [7] [8]. In the human-centered care model, the individual's disease differences, personality, and genome characteristics all determine the individualization and precision of nursing [9]. In general terms, it is the right patient to be taken accurate and reasonable care plans for the symptoms or clinical performance at the right time.

\section{System Construction}

\subsection{Build a Cancer Knowledge Network}

Current research shows that each cancer has its own unique genetic properties and tumor-specific markers, and common features of many types of cancer [10]. In the face of the challenge of cancer precision medicine, an individual-centered multilevel human disease knowledge integration database must be established. Based on this, a "cancer knowledge network" that can be used to accurately classify diseases is formed, as shown in Figure 1. It is used to guide clinical practice evidence, provide knowledge of health and disease awareness, and is used in many ways for health care workers and patients [11]. With the rapid development of digital networks and science technology, individual self-care ability has gradually increased. People can use their own genetic files to understand that they may be sick and choose their own medical services [12]. Through data self-management and prevention, we have developed cancer precision medicine that can provide each individual with the best medical care [13].

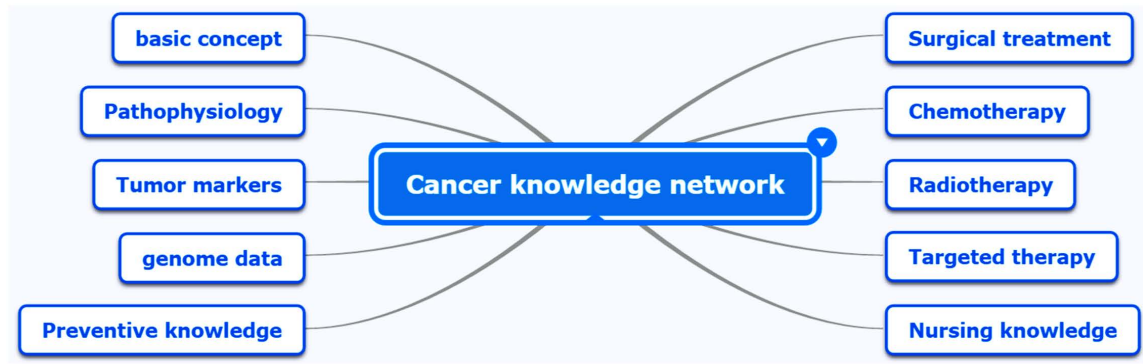

Figure 1. Cancer knowledge network. 


\subsection{Training Advanced Practice Nurses}

The primary task and important responsibility of nursing managers is not the manager but the development of nursing talent. In the current transformation of the precision medical model of cancer, nursing managers also need to change their management thinking and develop advanced practical nurses for cancer precision cancer nursing. Recent advances in tumor markers and biomedical technologies have begun to change the basic principles of cancer therapeutics and clinical trials. In order to design innovative adaptive tests, to develop accurate cancer treatment plans, and to provide appropriate, safe, and effective care, nursing staff are required to undergo standardized training, master relevant bio-marker knowledge, Malignant tumor-related molecular definitions and so on [14]. However genome testing also brings many ethical issues. Therefore, nurses need to understand the process of genome testing and the impact of the results and work collaboratively with the doctors [15]. In the process of clinical implementation of precise nursing, caregivers should maintain the right to be recruited and consent to participate in gene-spatial-related research, while also paying attention to the privacy of patients' health information and their willingness to use their personal health information. We do our best to provide the optimal care and maximize the benefits for precise treatment of cancer patients.

At the same time, in the study of Adams L, a retrospective study was performed on histology types and staging of non-small cell lung cancer and metastatic melanoma [16]. The study found that increasing patients' understanding of tumor genetics and molecular markers and expanding patients' treatment programs can often improve their quality of life. Nursing personnel understand the role of genetics and molecular markers in the diagnosis and treatment process, will be able to better assess or anticipate patient needs and challenges, and provide precision medical-related nursing education and decision support [17]. We need to carry out advanced practice nurses' practice, research, and education in genetics/genome to deepen advanced nursing practice research by identifying and implementing genome-based risk assessment, care, and management, and to contribute to the improvement of the genome health of all individuals and people [2] [18].

\subsection{Provide Clinical Decision Support System}

Researchers such as Vorderstrasse A.A. suggest that changes in nursing education, clinical decision support, and health systems are necessary during the process of supporting and promoting precision medicine and providing patients with personalized assessments, nursing, and treatment [19]. However, nurses play a key role in providing cancer precision nursing. The implementation of precise nursing interventions by advanced practice nurses can help elderly cancer patients recover faster and improve their quality of life [20] [21]. Secondly, the clinical decision support system for advanced practice nurses in oncology is worth studying, and the opportunity to implement interventions from genome is 
worth exploring. Developing clinical decision support tools is a complex health information processing technology. The clinical decision support tools can translate and integrate genome information and patient information, providing advice and evidence for personalized care [22]. However, our country's clinical decision support system can learn from the United States to establish a corresponding cancer knowledge network [23].

In 2011, the TeleDiaFoS surveillance system developed by the Polish scholar Ladyzynski proved to be very beneficial for the conversion of diabetic ulcer wound area into basic parameters [24]. In the same year, the Vanderbilt-Ingram Cancer Center successfully developed the My Cancer Genome system, which aims to provide clinical decision support for oncology nurses [22]. This is the first personalized cancer decision support tool in the United States, with data from nursing, medical literature, and the Internet. Based on this, smart medical care has been developed by embedding a variety of sensors in medical devices and using information networks for mobile health-care services. It realizes the exchange and seamless connection of various medical data, and carries out real-time dynamic monitoring and continuous tracking management of clinical nursing and treatment measures to assist nurses in making precise nursing health decisions [25].

\section{Clinical Application}

Clinical studies have shown that the adoption of precise nursing intervention model can effectively improve the care satisfaction of patients with advanced cervical cancer and help improve the quality of life of patients [26]. Therefore this method is worthy of clinical application and promotion. Psychological care, symptom management, medication management, and health education related to precise diagnosis are important aspects of the implementation of cancer precise nursing.

\subsection{Health Education}

The study points out that mastering the knowledge of systems biology improves the ability of nurses to implement evidence-based nursing practice and introduces patients and their families to new methods of diagnosis and treatment [27] [28]. Advanced practice nurses are an important part of the health-care system and they will be key implementer of cancer precise nursing [29]. Advanced practice nurses who have received professional training understand the process of collection, processing, analysis, and precise diagnosis and treatment of tumor-related specimens. They can inform patients about biological analysis risks and explain their prediction accuracy evaluation [30].

Rapidly developing genetics and genome are changing all aspects of cancer nursing and practice [31]. Advanced practice nurses can spread the knowledge of genetic genome to cancer patients and their families, and guide them to advocate evidence-based genetic genome as a guideline for treatment to reduce the 
risk of cancer or improve the outcome of cancer symptom management [32]. The cognitive needs of patients for precision medicine have also become the theoretical basis for nurses to implement precision nursing measures for individuals.

\subsection{Medication Management}

Different tumor patients have different treatment responses to different chemotherapy drugs. The range of safety doses for anticoagulant drugs used in patients with thrombosis and toxic drugs used for patients with advanced cancer pain is too narrow. And many factors have caused precise doses to become a great challenge for clinical drug safety. The implementation of special management of medical and nursing integration for drug administration and genetic testing can help medical personnel and patients accurately manage the dosage and its side effects [12].

\subsection{Psychological Care}

Cancer not only seriously harms human health, but also brings great psychological pressure and mental stress to cancer patients. The tedious nursing work may lead nurses to focus on the physiological needs of cancer patients, such as analgesics and pain care treatment programs based on each patient's genetic code and pain sensitivity [33], while neglecting the patient's psychological needs. Cancer precise nursing not only requires precise nursing for precise targeted therapies, but also requires precise nursing of cancer patient's emotional and psychological. In the past, nurses usually used listening, documentary, and especially various types of scales to assess the patient's physical state or mental state [34]. However, precision nursing is the analysis of the patient's gene or gene spectrum, and accurate assessment of the patient's physiological changes or mental state from the molecular biology level. Thus, appropriate patients can be given appropriate care at the right time and truly precision nursing, which can vary from person to person, from case to case, from treatment to treatment.

\subsection{Symptom Management}

The principle of treatment for most cancer patients is based on surgical treatment, supplemented by chemotherapy and radiotherapy. Patients can resolve problems in a timely manner through the nurse's precise nursing during the hospital, but most patients still have varying degrees of health problems or potential risks of complications after discharge. Because patients and their family members lack awareness of these symptoms, they often delay the best time for complications and other treatments [35]. So this requires nursing staff to extend precision care to the family. Research shows that continuing nursing may not improve the survival of gynecological poorly differentiated cancer patients [36]. However, focusing on patient needs and mental health, based on the patient's gene or gene spectrum analysis, predicting problems, providing timely nursing 
interventions, providing precise nursing can avoid causing patients with insecurity and anxiety, and improving the relationship between nurses and patients [36]. In the process of continuous nursing, the nursing staff performs accurate phenotype or deep phenotype analysis of cancer patients, strengthens symptom management of patients, and implements precise nursing interventions so that the patient's body is in the best state of recovery, which may improve the outcome of the prognosis.

\section{Summary}

In view of the rapid transformation of the medical model, the development of cancer precise nursing in our country is now in its infancy. We must not blindly equate "precision medicine" with "individualized medicine", nor can we confine ourselves to genome sequencing. Similarly, "cancer precise nursing" cannot be simply equated with "cancer specialist care" or "personalized care". The basic principles and guidelines for nursing work are people-oriented. As shown in Figure 2, the key to establishing a modern cancer nursing work model lies not only in the establishment of a highly humane nursing team, but also in the establishment of a team of highly qualified and precise nursing personnel. The transition from personalized care to precision nursing is the foundation for the continuous improvement of clinical work and research in cancer care.

As China is a critically affected area for cancer, Chinese nurses actively explore and develop precision nursing in the fight against cancer. It will become an important principle for future nursing practice. It is recommended that researchers should support gene therapy-based nursing practice, research and education, actively carry out research on clinical practice of cancer precision nursing, and promote the combination and sharing of gene spectrum information to build knowledge and ability of nursing staff to accurately care for cancer, and to promote the development of cancer precision nursing in China.

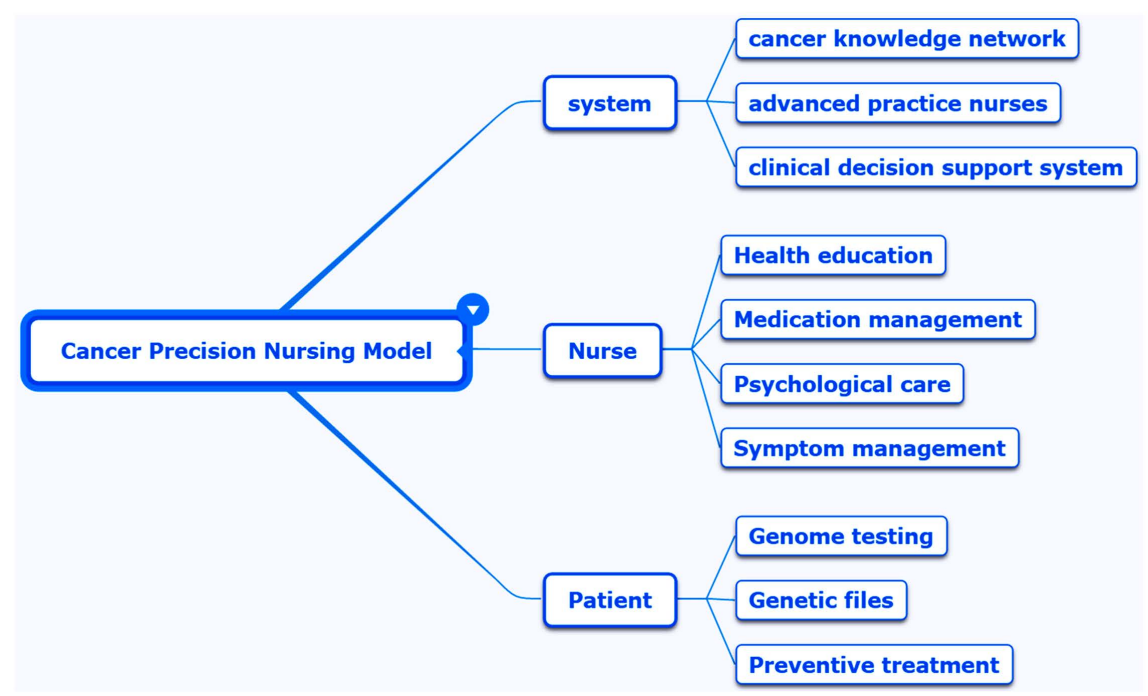

Figure 2. Cancer precision nursing model. 


\section{Acknowledgements}

This study was supported by Hubei Natural Science Fund Project (key project, 2017CFB786).

\section{Conflicts of Interest}

The authors declare no conflicts of interest regarding the publication of this paper.

\section{References}

[1] Fu, M.R., Tian, Y.L., Hu, X.Y., et al. (2017) Application Fields and Development Direction of Precision Nursing. Chinese Journal of Nursing, 10, 1273-1275.

[2] Williams, J.K., Katapodi, M.C., Starkweather, A., Badzek, L., Cashion, A.K., Coleman, B., et al. (2015) Advanced Nursing Practice and Research Contributions to Precision Medicine. Nursing Outlook, 64, 117-123.

https://doi.org/10.1016/j.outlook.2015.11.009

[3] Yan, Q.Y. (2016) Challenges of Nursing Disciplines in the Context of Precision Medical Care. Nursing Research, 30, 3713-3716.

[4] Wujcik, D. (2016) Scientific Advances Shaping the Future Roles of Oncology Nurses. Seminars in Oncology Nursing, 32, 87-98. https://doi.org/10.1016/j.soncn.2016.02.003

[5] The White House (2015) Fact Sheet: President Obama's Precision Medicine Initiative.

https://obamawhitehouse.archives.gov/the-press-office/2015/01/30/fact-sheet-presi dent-obama-s-precision-medicine-initiative

[6] Yuan, C. (2015) Precision Nursing: New Era of Cancer Care. Cancer Nursing, 38, 333-334. https://doi.org/10.1097/NCC.0000000000000290

[7] Suhonen, R., Papastavrou, E., Efstathiou, G., et al. (2011) Nurses' Perceptions of Individualized Care: An International Comparison. Journal of Advanced Nursing, 67, 1895-1907. https://doi.org/10.1111/j.1365-2648.2011.05626.x

[8] Munro, C.L. (2015) Individual Genetic and Genomic Variation: A New Opportunity for Personalized Nursing Interventions. Journal of Advanced Nursing, 71, 35-41. https://doi.org/10.1111/jan.12552

[9] Lu, J.F., Liu, Y.L., Cao, Z.H., et al. (2015) Principles of Combination of Evidence-Based Medicine and Individualized Treatment in Clinical Teaching of Chronic Hepatitis B. Beijing Medicine, 12, 1201-1202.

[10] Mericbernstam, F., Farhangfar, C., Mendelsohn, J. and Mills, G.B. (2013) Building a Personalized Medicine Infrastructure at a Major Cancer Center. Journal of Clinical Oncology, 31, 1849-1857. https://doi.org/10.1200/JCO.2012.45.3043

[11] Sheng, Y. and Zhang, Y. (2016) The Development of Clinical Decision Support System in U.S. and Its Enlightenment. Chinese Journal of Health Information Management, 13, 257-261.

[12] Corwin, E.J. and Ferranti, E.P. (2016) Integration of Biomarkers to Advance Precision Nursing Interventions for Family Research across the Life Span. Nursing Outlook, 64, 292-298. https://doi.org/10.1016/j.outlook.2016.04.007

[13] Wu, J.R. (2015) Precision Medicine Based on Systematic Biology. Life Science, 5, 558-563.

[14] Heckmanstoddard, B.M. and Smith, J.J. (2014) Precision Medicine Clinical Trials: 
Defining New Treatment Strategies. Seminars in Oncology Nursing, 30, 109. https://doi.org/10.1016/j.soncn.2014.03.004

[15] Lemoine, C. (2014) Precision Medicine for Nurses: 101. Seminars in Oncology Nursing, 30, 84. https://doi.org/10.1016/j.soncn.2014.03.002

[16] Adams, L. (2014) Precision Medicine in Oncology Standard of Care. Seminars in Oncology Nursing, 30, 100-108. https://doi.org/10.1016/j.soncn.2014.03.003

[17] Richmond, E.S. and Dunn, D. (2012) Biomarkers: An Overview for Oncology Nurses. Seminars in Oncology Nursing, 28, 87-92. https://doi.org/10.1016/j.soncn.2012.03.002

[18] Maki, K.A. and DeVon, H.A. (2018) A Nursing Theory-Guided Framework for Genetic and Epigenetic Research. Nursing Inquiry, 25, e12238. https://doi.org/10.1111/nin.12238

[19] Vorderstrasse, A.A., Hammer, M.J. and Dungan, J.R. (2014) Nursing Implications of Personalized and Precision Medicine. Seminars in Oncology Nursing, 30, 130-136. https://doi.org/10.1016/j.soncn.2014.03.007

[20] Bai, X.H., Zang, S. and Su, L.R. (2012) The Effect of Personalized Nursing Model in Radiotherapy Care for Elderly Cancer Patients. China Medical Herald, 27, 141-143.

[21] Oruche, U.M., Carpenter, J.S., Renbarger, J. and Ross, S.E. (2016) Raising the Level of Nursing Involvement in the National Precision Medicine Initiative: An Example. Journal of Child and Adolescent Psychiatric Nursing, 29, 85-88. https://doi.org/10.1111/jcap.12141

[22] Carney, P.H. (2014) Information Technology and Precision Medicine. Seminars in Oncology Nursing, 30, 124-129. https://doi.org/10.1016/j.soncn.2014.03.006

[23] Yang, C.H., Wang, T.J., Huang, S.M., Liu, N. and Wang, Y. (2016) Foreign Clinical Decision Support System Supporting Precision Medicine. Chinese Journal of Medical Library and Information Science, 25, 14-19.

[24] Ladyzynski, P., Foltynski, P., Molik, M., Tarwacka, J., Migalskamusial, K., Mlynarczuk, M., et al. (2011) Area of the Diabetic Ulcers Estimated Applying a Foot Scanner-Based Home Telecare System and Three Reference Methods. Diabetes Technology \& Therapeutics, 13, 1101-1107. https://doi.org/10.1089/dia.2011.0082

[25] Zhu, L. (2015) Prospects for the Application of Intelligent Nursing in Hospital Care Services. Journal of Practical Clinical Medicine, 19, 208-209.

[26] Chang, S., Zhao, J. and Chen, J.S. (2017) Application and Observation of Precise Nursing Service Model in Anxiety Symptoms of Patients with Advanced Cervical Cancer. Chinese Health Nutrition, 14, 4-5.

http://g.wanfangdata.com.cn/details/detail.do?_type=perio\&id=zgbjyy-kp20171400 $\underline{4 \#}$

[27] Schallom, L., Thimmesch, A.R. and Pierce, J.D. (2011) Systems Biology in Critical-Care Nursing. Dimensions of Critical Care Nursing, 30, 1. https://doi.org/10.1097/DCC.0b013e3181fd0169

[28] Founds, S. (2018) Systems Biology for Nursing in the Era of Big Data and Precision Health. Nursing Outlook, 66, 283-292. https://doi.org/10.1016/j.outlook.2017.11.006

[29] Chadwell, K. (2013) Clinical Practice on the Horizon: Personalized Medicine. Clinical Nurse Specialist, 27, 36. https://doi.org/10.1097/NUR.0b013e318277703c

[30] Neumann, R.M., Garvey, C. and Kaufman, S. (2014) Biospecimen Collection, Processing, and Analysis: New Challenges for Oncology Nurses. Seminars in Oncology Nursing, 30, 117-123. https://doi.org/10.1016/j.soncn.2014.03.005 
[31] Calzone, K.A., Kirk, M., Tonkin, E., Badzek, L., Benjamin, C. and Middleton, A. (2018) The Global Landscape of Nursing and Genomics. Journal of Nursing Scholarship, 50, 249-256. https://doi.org/10.1111/jnu.12380

[32] Santos, E.M., Edwards, Q.T., Floriasantos, M., Rogatto, S.R., Achatz, M.I. and Macdonald, D.J. (2013) Integration of Genomics in Cancer Care. Journal of Nursing Scholarship: An Official Publication of Sigma Theta Tau International Honor Society of Nursing, 45, 43-51. https://doi.org/10.1111/j.1547-5069.2012.01465.x

[33] Manworren, R.C.B. (2015) Multimodal Pain Management and the Future of a Personalized Medicine Approach to Pain. Aorn Journal, 101, 307-318. https://doi.org/10.1016/j.aorn.2014.12.009

[34] Xu, M.K., Gaysina, D., Barnett, J.H., Scoriels, L., Lagemaat, L.N.V.D., Wong, A., et al. (2015) Psychometric Precision in Phenotype Definition Is a Useful Step in Molecular Genetic Investigation of Psychiatric Disorders. Translational Psychiatry, 5, e593. https://doi.org/10.1038/tp.2015.86

[35] Qin, G.R. (2012) Current Status and Development Trend of Extended Care for Discharged Patients. Journal of Nursing, 27, 89-91.

[36] Dahl, L., Wittrup, I., Petersen, L.K., Blaakaer, J. and Væggemose, U. (2015) Paradoxes of Follow-Up-Health Professionals' Views on Follow-Up after Surgical Treatment in Gynecological Cancer. Acta Oncologica, 54, 194-199.

https://doi.org/10.3109/0284186X.2014.935475 